\title{
Segmental definition: Part II. Application of an indirect method in osteopathic manipulative treatment
}

\author{
WILLIAM L. JOHNSTON, DO, FAAO \\ East Lansing, Michigan
}

Part I of this series showed how somatic function is examined by application of a series of passive gross motion tests. In osteopathic manipulative treatment of somatic dysfunction, diagnosis of the asymmetries in resistance to segmental motion tests provides a basis for the methods the physician applies and criteria for posttreatment evaluation. Successful use of the method described produces a return to symmetry in posttreatment motion tests not only at the specific segmental asymmetry being addressed, but also at the adjacent segments where mirror image asymmetries had once prevailed. It appears that, within this three-segment dysfunctional unit, a sensory input of somatic origin from the primary central dysfunction accounts for the mirror image asymmetries at adjacent segments. This behavior implies an adaptive reflex basis that is somatosomatic. Experience in comparing responses to motion tests throughout the three-segment unit can provide greater accuracy in segmental diagnosis and internal validation of the location of the primary dysfunctional segment where osteopathic manipulative treatment is more effective.

Defining the motion characteristics of segmental dysfunction provides a basis for most specific osteopathic manipulative approaches. Palpable tissue texture and structural position have also been important factors influencing manipulative techniques. However, the diagnosed limitations in mobile function, represented by the directions of motion restriction, have provided palpable cues about specific directions of force to be applied in treatment.

When the incremental aspects of these cues are appreciated ${ }^{1}$ as an immediately increasing resistance toward a sense of barrier in one direction, and an immediately increasing ease toward a sense of potential release in the opposite direction, then the terms ${ }^{2}$ direct (toward the sense of resistance) and indirect (away from the sense of resistance) offer a classification of osteopathic manipulative procedures based on the diagnosed asymmetry to be addressed. Thus, the examiner can move from the reception of diagnostic cues to their prompt application in treatment.

(The term "combined technique" has been applied to a few procedures in which there is recognized use of some directions of increasing ease to produce initial relaxation of tissues and some directions of force to then overcome the remaining resistance.)

Many terms have been used in the past to try to describe osteopathic manipulative procedures that apply these two methods. For direct techniques, the following expressions were summarized from the literature by Lippincott ${ }^{3}$ : taking out the slack; thrusting force; high-velocity, low-amplitude; correctional pop; joint locking; teasing/rocking; poising the joint on the brink of correction; pressure in the direction toward its normal situation; disengaging the articular surfaces, either by separation or approximation (to reseat themselves). Conceptually, the restricted motion usually represented "the 
problem." Treatment was in a direct fashion to overcome that restriction and restore mobility.

For indirect techniques, the following expressions have been used ${ }^{3,4}$ : exaggerating the lesion position; balancing the ligamentous tension; position of ease; farther in the direction toward its position of strain; release by positioning. Here, the conceptual basis often involved the position of one bone on the bone below. Treatment consisted of adjusting the malposition to find the best new positional relationship as indicated by the palpable sense of decreasing tension and improved balance in the surrounding soft tissues.

The following description of osteopathic manipulative procedure presents an example of the application of an indirect method only. It has been termed functional technique $e^{5,6}$ because it makes use of information gained about motion function, by monitoring physiologic response at individual segments to passive, gross motion tests. The physician temporarily sets aside positional concepts and palpatory information about joint position in favor of a continuous feedback, during motion, of positive palpatory cues about trends toward improving motion function at a dysfunctional segment (spinal or appendicular). During examination, the physician compares the segment's immediate compliance with, or resistance to, opposing directions of each passive, gross movement test. Directions of motion then introduced in treatment are those that lead to an increasing sense of compliance (decreasing sense of resistance) of that segment. These statements establish the dysfunctional segmental part as a focus for manipulation, and specify a method to be applied; they describe the diagnostic information to be sought, and express guidelines for applying specific directions of motion during the manipulative procedure.

\section{Procedure}

In planning a functional approach to osteopathic manipulative treatment, attention should be paid to the following criteria for successful outcome:

1. Introduction of motion in any one direction is initial only, with minimal forces applied.

2. Motion directions are toward a sense of immediately increasing ease (manifested by a decreasing sense of resistance to pressure at fingers monitoring response at the dysfunctional segment).

3 . Single elements of rotary and translatory directions are combined, effecting the control of an eventual torsion pathway for body movement.

4. Response at the dysfunctional segment to an active respiratory component is also monitored.

5 . The operator follows a continuous feedback signal of increasing ease (decreasing tissue resistance)

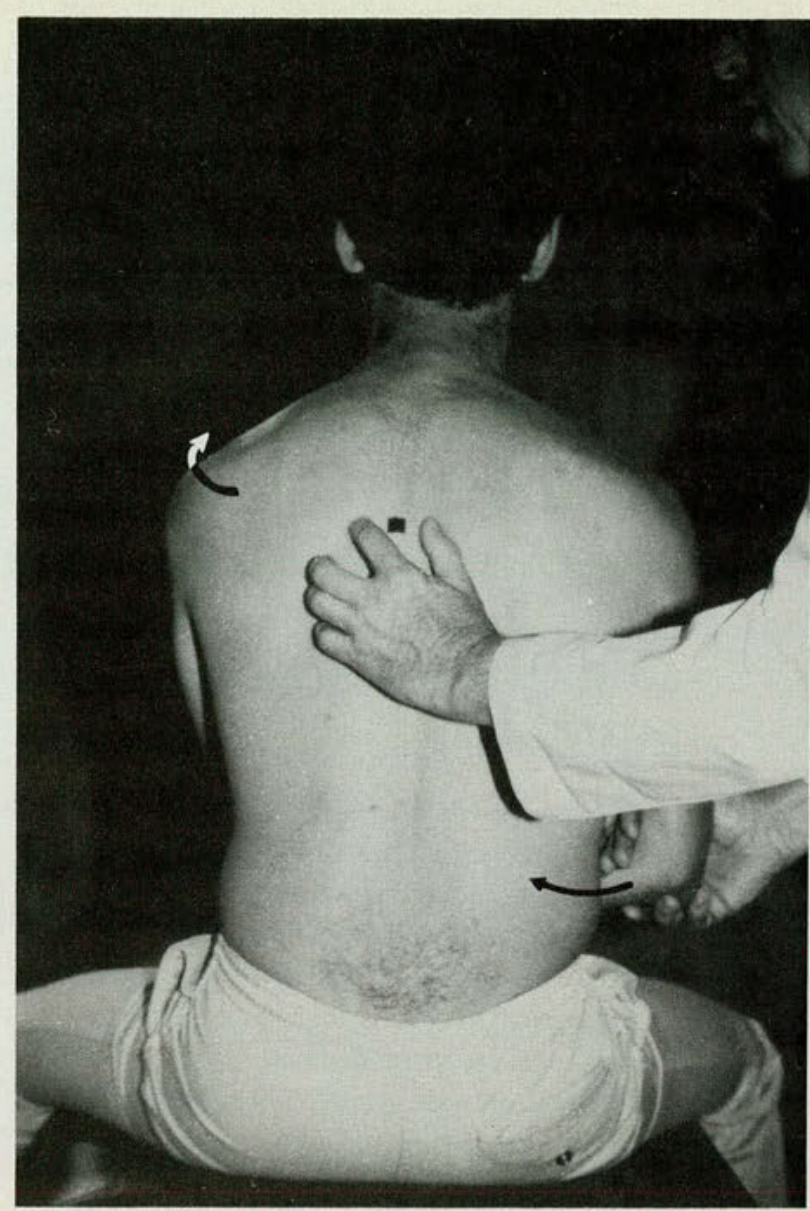

Fig 1. Examiner's left fingers, overlying T6, monitor segmental response to introduction of shoulder and trunk rotation to the right.

throughout the procedure.

Successful outcome accomplishes a sensed release of the segmental tissues' holding forces, and then a free return to resting position without encountering any further palpable sense of increasing resistance. The segment's new functional symmetry attained is evident in responses to motion retesting. Time for the testing, treatment, and retesting to be detailed is about $2 \mathrm{~min}$.

The example of functional technique presented here will build on the circumstances that were introduced in Part I of this paper. The subject is seated (Fig. 1). There is a primary dysfunctional segment at T6, with segmental tissues showing immediately increasing resistance to rotation right of the shoulders and trunk. The accompanying resistance to rotation left at T5 and T7 is tested by reversal of hand placements and comparison with the increasing ease in rotation left at T6.

In Fig. 2, sidebending (lateral flexion) is being tested to the right. Comparison of the response with that to sidebending left (with hand placements re- 

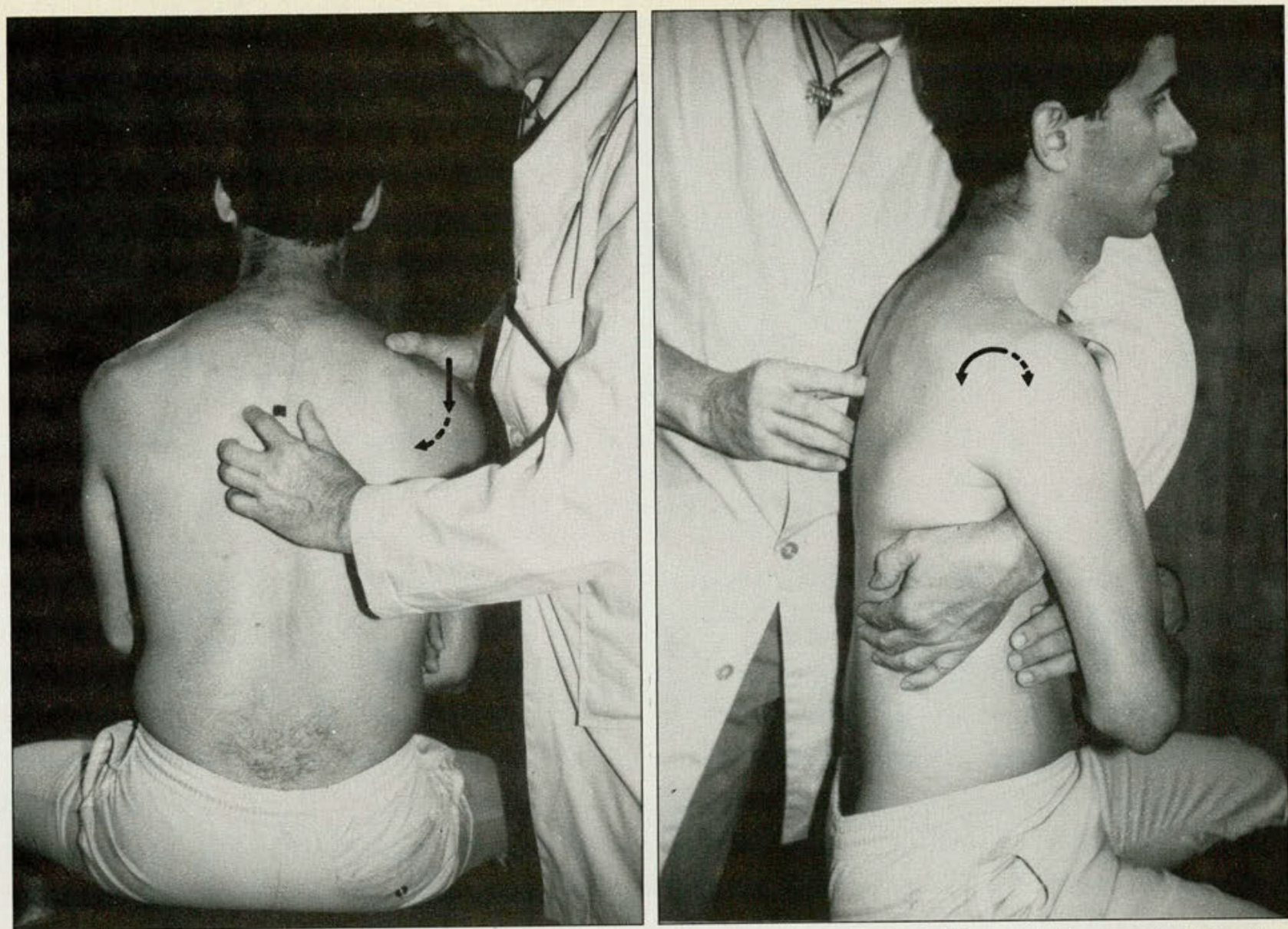

Fig 2. Examiner's right hand on subject's right shoulder introduces a moderate caudally directed force to initiate trunk sidebending (lateral flexion) to the right. Fig 3. Examiner control of subject's shoulders and trunk in this position enables introduction of extension and then forward flexion of motions (indicated by arrows); responses are monitored at operator's right hand contacting at T6 level.

versed) shows increasing tissue resistance to sidebending right and increasing ease during sidebending left. Testing of sidebending left with hand contact at T5 and then at T7 demonstrates increasing resistance to sidebending left at both segments.

In Fig. 3, segmental tissue compliance is being compared during initiation of flexion/extension and then forward flexion. There is increasing ease in extension, compared to the increasing resistance to flexion. The operator now has sufficient information (from our example of findings) to plan the eventual positioning of subject and self for application of these three specific components in the treatment phase. As indicated in Fig. 4, the operator's left arm position, over the left shoulder and under the right, will allow easy introduction of forces to initiate sidebending left, rotation left, and extension, in a smooth torsional arc. Each of these directions will begin to diminish the local tissue resistance being monitored at $\mathrm{T} 6$ throughout the manipulative procedure. Note that the operator's body approximates the subject's thorax for easy control of the movement to be introduced; with the operator's legs semiflexed, even slight changes in position of the operator's torso will initiate specific directions of movement of the subject with careful control and minimal force.

Once positioned, the operator can now make decisions during testing of translatory directions of movement. Using slight shifting forces to control movement of the subject's body, the examiner can compare segmental responses at $\mathrm{T} 6$, first during motion to left with right (Fig. 5), then posterior anterior movement (Fig. 6), and then with cephalad lifting with slight caudad compression (Fig. 7). Such testing reveals increasing ease during movements in left, anterior, and cephalad directions, compared with increasing resistance during movement in right, posterior, and caudad directions. Finally, the operator monitors a moderately increased range of respiratory excursion to compare the initial response at $\mathrm{T} 6$ to the demands for inhalation versus exhalation movements. The segmental tissues indicate increasing compliance to inhalation with in- 

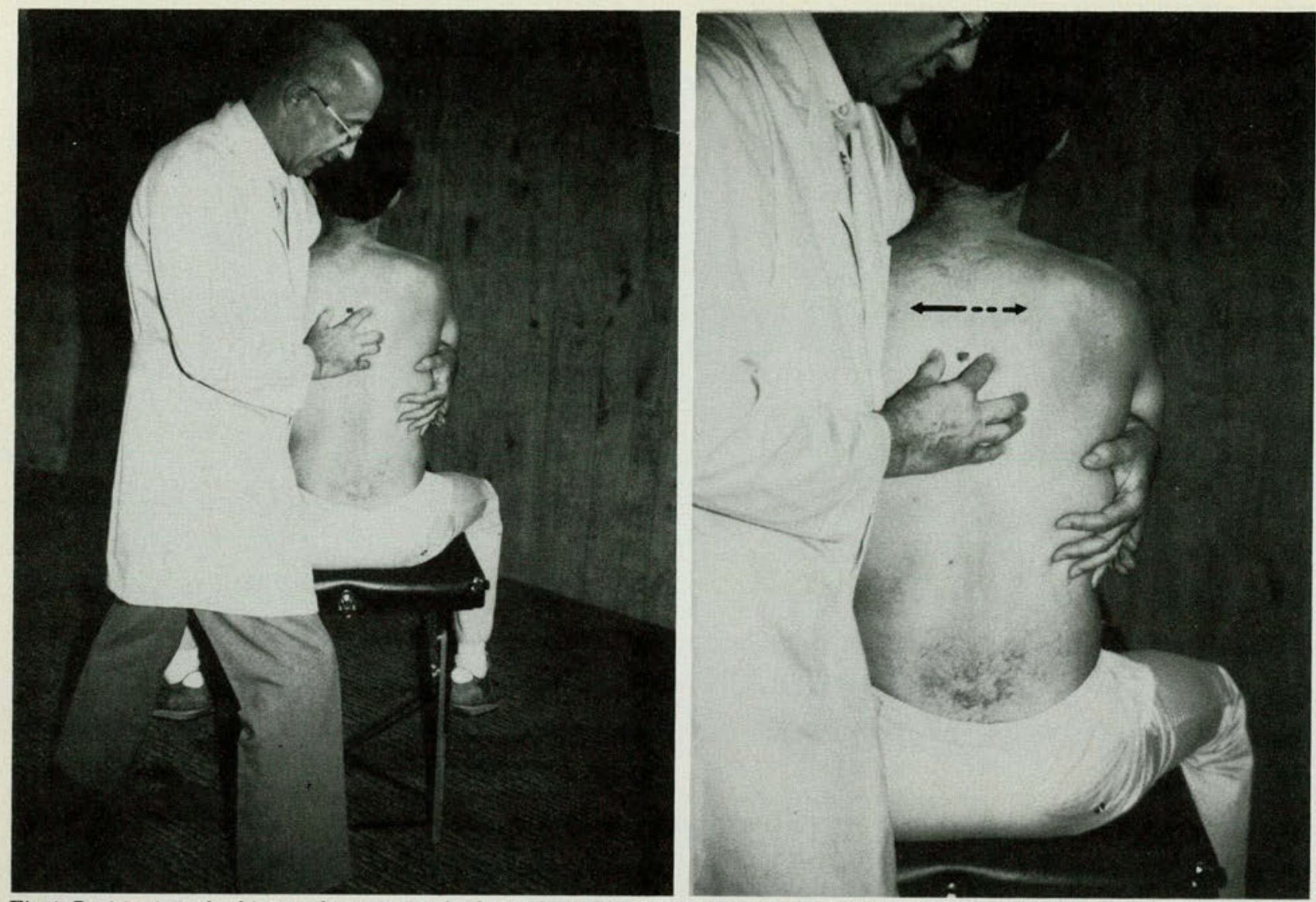

Fig 4. Positioning of subject and operator to facilitate the initial introduction of three rotary components already tested, with increasing compliance in extension, sidebending left, and rotation left. Fig. 5. Testing lateral translatory directions of movement to left and then right as indicated by arrows.

creasing resistance during exhalation. (Though not specified throughout this description, each asymmetry diagnosed at T6 is accompanied by opposing asymmetries at $\mathrm{T} 5$ and $\mathrm{T} 7$, when the responses to motion directions are compared at these adjacent segments.)

The three rotary components of sidebending left, rotation left, and extension are then combined in the initiation of a smooth torsional arc of body motion as shown in Fig. 8, translating to the left, anterior, and cephalad. The subject is asked to begin a slow inhalation, and the accumulating ease is carefully monitored. Often, even during the single inspiratory phase, continuing fine adjustments of directional controls by the operator will effect an optimal reduction and then release of tissue resistance. The palpable sense of release appears to be a final yielding of the resistance formerly evident in the opposing direction. It prompts the operator to control the return of the patient to a central resting position, while the segment to assure tissue compliance throughout the return.

Once resolution is complete, retesting directions of motion previously restricted at $\mathrm{T} 6$ shows no sign of any remaining tissue resistance to initial movements. Further retesting at T5 and T7 reveals that these adjacent segments also show a return to symmetry of tissue compliance in response to initial aspects of all motion tests.

\section{Discussion}

Historically, the concept of symmetry in function of a mobile segment has been central to the classification of osteopathic manipulative techniques as direct and indirect. The underlying principle being addressed in osteopathic manipulative treatment involves the relation between somatic dysfunction and departures from health. Symmetry in segmental function is a standard of normal behavior during passive motion tests. The asymmetries in segmental dysfunction-asymmetric position of structures, increased and asymmetric muscular tension, and limited and asymmetric mobility during passive motion tests - constitute a departure from healthy function within the mobile system. Further, it is clinically recognized that restoration of 
symmetric function provides a positive direction in health care. The extent of the relationship between somatic dysfunction and the body's other systemic functions/dysfunctions is yet to be documented carefully. New knowledge about these systemic interrelationships awaits controlled clinical studies that include (1) tests for the presence of segmental asymmetry during physical examination and (2) a report of the findings of segmental dysfunction in the patient who is ill.

\section{Development of skill}

Local resolution of mobile asymmetry at a dysfunctional segment is a realizable goal during a single encounter for health care. While learning to apply an indirect method in osteopathic manipulative treatment, however, physicians may find that several attempts are required to perfect the best combination of movements and to learn the amounts of each that are needed to completely resolve the asymmetry. While the procedure is being perfected, varying degrees of release of the holding forces at a dysfunctional segment may take place. After return to a central resting position, retesting may reveal tissue resistance of reduced intensity, but sufficiently palpable to warrant further attention. Seldom does the diagnosed pattern of asymmetry alter during this process; rather, it is the examiner's level of skill that changes, improving in the ability to promote a maximum release of tissue resistance and symmetric response on reexamination. Range is not as critical an issue in functional technique as the control of specific directions in which tissue resistances decrease. In Fig. 9A, where axial rotation is used as an example for each component direction, the potential for increasing ease at the dysfunctional segment at $X 1$ is indicated early in the range of rotation left from point $X 1$ toward $X-1 L$. This decreasing tension begins to level off (for each component) as range increaseshere, as axial rotation continues beyond $X-1 L$. (A normal barrier sense begins to become evident as the end of range is approached in that direction to the left.) Combining all specific directions of motion during their initial introduction promotes the most rapid functional change toward the release of resistance at the segment.

The active respiratory component deserves separate comment, because of its contribution to success in this method of osteopathic manipulative treatment. Although respiration has often received attention in osteopathic manipulative techniques, it seldom has been used as a specific active motion test, except in evaluating mobility of ribs. As an active movement to bring about elevation and depression of rib structures, however, respiratory ex-

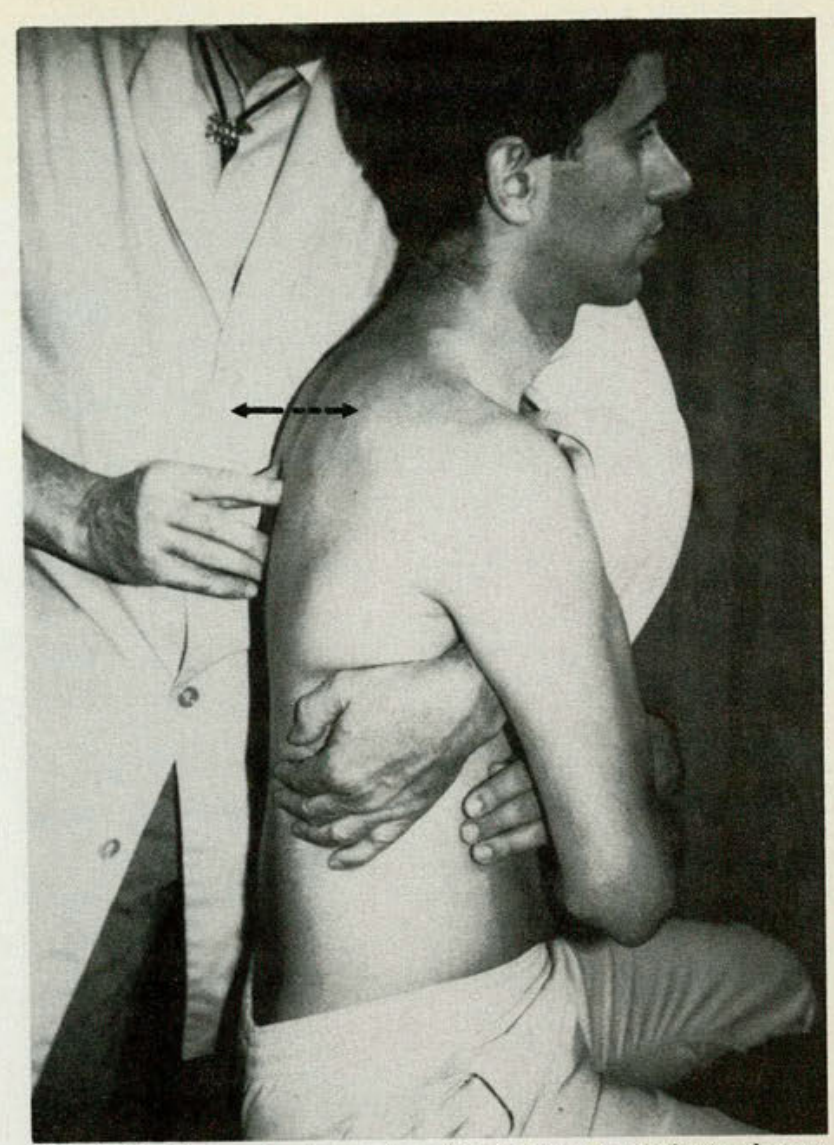

Fig. 6. Testing posterior and anterior directions of translatory movement.

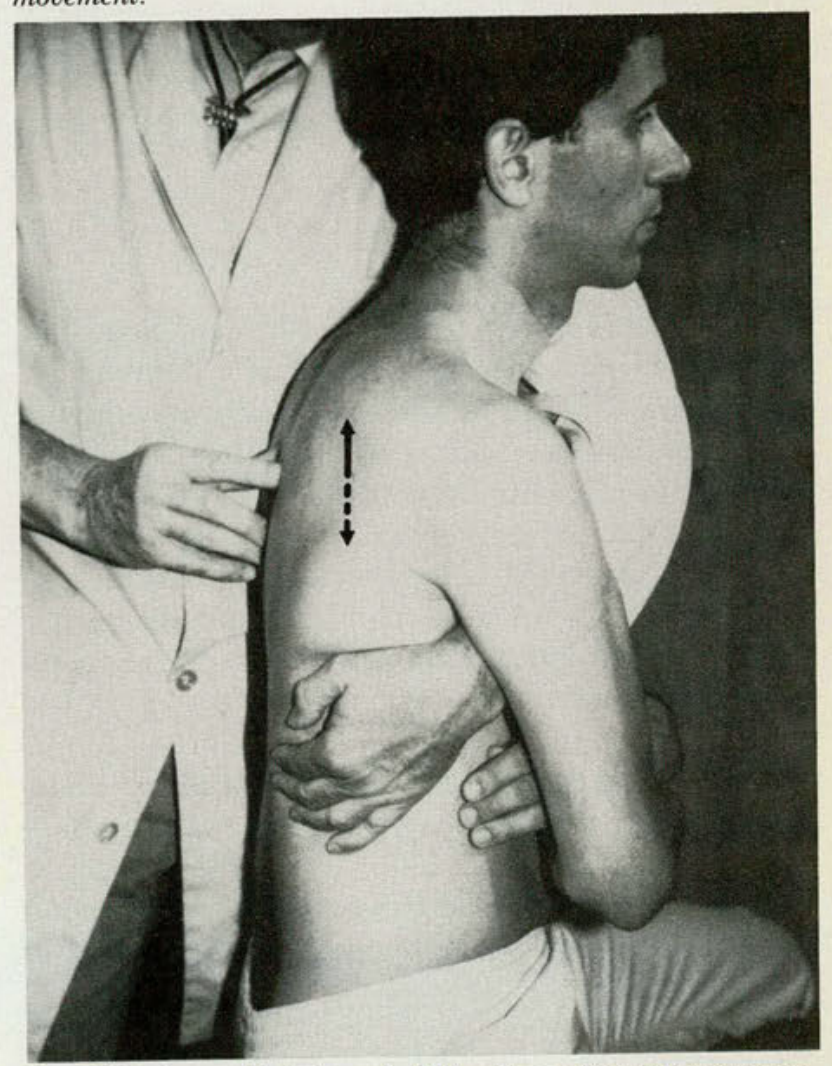

Fig. 7. Testing cephalad-caudad directions of translatory movement. 


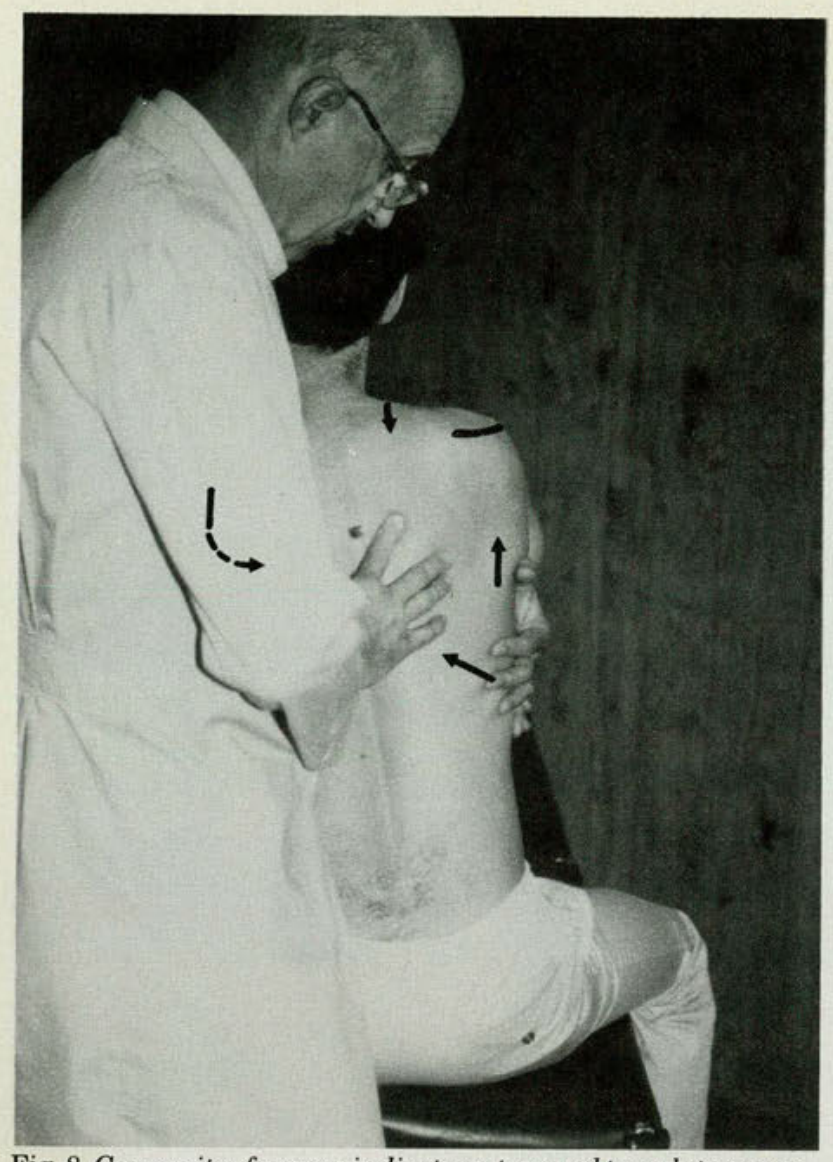

Fig. 8. Composite of arrows indicates rotary and translatory component directions, as each contributes to a torsional pathway for increasing compliance monitored by examiner's right hand contacting at T6 level. Component directions are indicated by arrows and include sidebending left, axial rotation left, extension, and translation cephalad; the straight arrow for translation left is angled to combine translations left and anterior.

cursion is not confined to the thoracic cage, but rather pervades the entire body as a demand for movement. Inhalation and exhalation movements may encounter compliance or resistance, the choice depending on the function/dysfunction of the body's normally mobile segments.

When a segment is dysfunctional, as indicated at $X 1$ in Figure $9 \mathrm{~A}$, it responds in an asymmetric fashion to the opposing phases of the active respiratory excursion: there is increasing compliance with one; increasing resistance to the other. Monitored as an active motion test, respiratory excursion provides another specific descriptor of the segmental asymmetry. As indicated in the procedure section, it also helps during treatment to bring about increasing ease and successful resolution of the asymmetry.

\section{Concepts of segmental reflex control}

The question of how the restricted motion is over- come with so little force is frequently raised. This question emphasizes only one aspect of the disturbed behavior-restriction-rather than the increasing-decreasing resistance curves evident during motion testing. The latter implies somewhat different physiologic aspects of the sensed restriction and provides a different opportunity for resolving the clinical dysfunction. In the example of functional technique given here, specific directions of motion become the major influence for change, not force. At the moment when release of resistance forces is sensed, the response (conceptually) appears to be the result of a matching in movement function, in which the local segmental control beccomes appropriate to the current, overall movement-a matching of adequacy in physiologic response to specific motion demand. The return to local controlled compliance of the mobile segment within the whole complex movement restores the opportunity for adequate part-to-whole functional relations of this segment within the mobile system.

The observed return to symmetry of function at the adjacent segments (T5, T7), after treatment that is focused for its clues at T6, requires comment about the nature of the three-segment unit of dysfunction. It appears that the adjacent segmental asymmetries represent a functional adaptation to a primary motion defect at $\mathrm{T} 6$; when that primary defect is resolved, the need to adapt is removed. The association of primary and secondary findings before treatment with the integrated responses after osteopathic manipulative treatment has become a predictable clinical phenomenon. A somato-somatic reflex basis for this segmental behavior is a logical consideration.

Basic knowledge about proprioceptor and nociceptor stimuli as a source of reflex communication from somatic tissues to other somatic tissues ${ }^{7}$ is well established. Even at rest, in response to only gravity and positional demands, the palpable findings of bony irregularity and increased resistance of muscular tissue to pressure at dysfunctional segments reflect ongoing stimulation of proprioceptive sensors. During movement, these palpable cues to the traffic on afferent pathways are highly erratic, since in some directions of ease they decrease whereas in opposing directions of resistance they increase. As indicated in Figure 9A, each direction initiates an increment of change from $X 1$ toward $X L$ during initial rotation left, and from $X 1$ to $X 1-R$ during rotation right. This continuum of change in tissue tone is in contrast to the low level of change seen in Figure 9B between $X-L$ and $X$ $R$, referred to as a dynamic neutral..$^{5}$ The sheer immediacy of the changes palpated during motion test- 


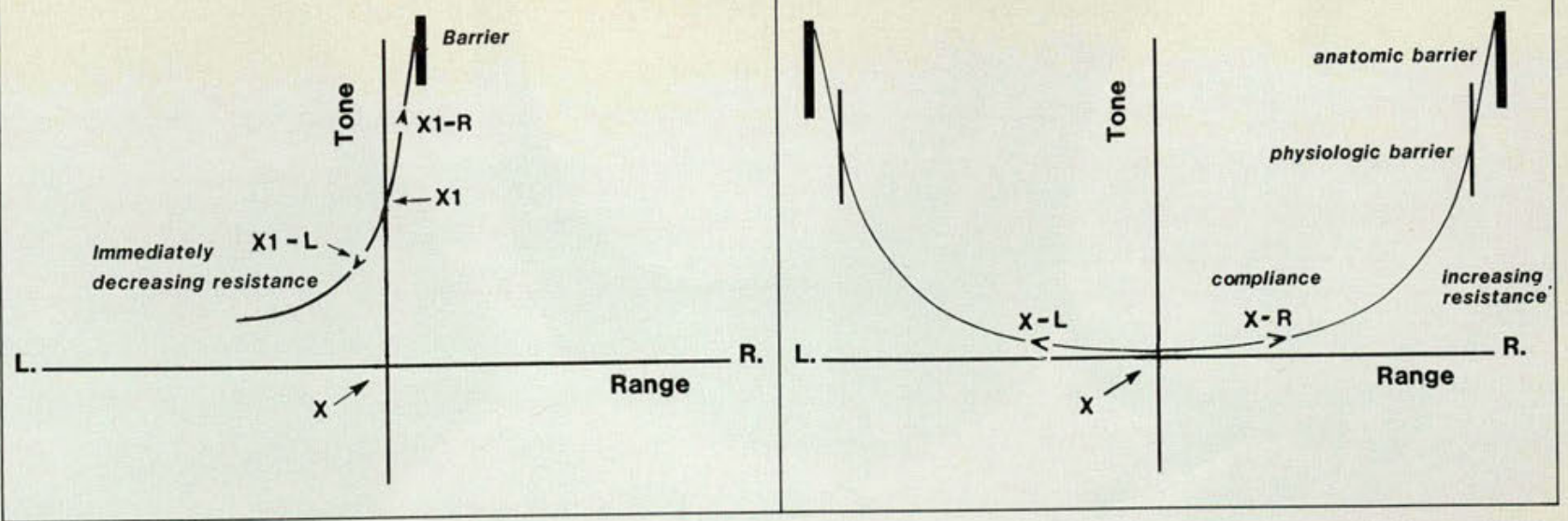

Fig. 9A. Schematic representation for asymmetric behavior at a dysfunctional segment (only axial rotation is indicated). The inclined line X1 through X1-L shows initial early range of motion during which increments of ease (decreasing resistance) are available during functional manipulation. Fig. 9B. Schematic representation for symmetry at a nonlesioned segment (only axial rotation is indicated). The line $X-L$ through $X-R$ indicates initial early range of motion where symmetry in response to opposing directions of motion reflects low level of change in palpable resistance to pressure (dynamic neutral).

ing and treatment suggests the moment-to-moment afferent monitoring by numerous muscle spindle stretch receptors and the resulting efferent control of muscle contraction/relaxation as a physiologic basis for interpreting the response to osteopathic manipulation being reported here.

1. Johnston WL: Segmental definition, Part I: A focal point for diagnosis of somatic dysfunction. JAOA 1988;88:99-105.

2. Hoover HV: Fundamentals of technic. AAO Year Book, 1949, pp 2541.

3. Lippincott HA: Basic principles of osteopathic technique. AAO Year Book, 1961, pp 45-48.

4. Jones LH: Spontaneous release by positioning. The DO 1964;4:109116.

5. Bowles CH: A functional orientation for technic: A tentative report on a functional approach to specific osteopathic manipulative problems developed in the New England Academy of Applied Osteopathy during 1952 1954. AAO Year Book, 1955, pp 177-191.

6. Hoover HV: Functional technic. AAO Year Book, 1958, pp 47-51.

7. Henneman E: Organization of the spinal cord and its reflexes in Mountcastle VB (ed): Medical Physiology. Ed. 14. St. Louis, CV Mosby Co, 1980, vol 1, pp 762-86.

Dr. Johnston is professor, Department of Family Medicine, Michigan State University - College of Osteopathic Medicine, E Lansing, Mich.

Dr. Johnston, MSU-COM, W Fee Hall, E Lansing, Mich 48824. 
